\title{
Proposal for a standardised treatment regimen to manage pre- and extensively drug-resistant tuberculosis cases
}

\author{
Jose A. Caminero ${ }^{1,2}$, Alberto Piubello ${ }^{2,3}$, Anna Scardigli ${ }^{4}$ and \\ Giovanni Battista Migliori $\mathbb{1}^{5}$
}

Affiliations: 'Pneumology Department, Hospital General de Gran Canaria “Dr. Negrin”, Las Palmas de GC, Spain. ${ }^{2}$ Tuberculosis Division, International Union against Tuberculosis and Lung Disease (The Union), Paris, France. ${ }^{3}$ MDR-TB Division, Damien Foundation, Brussels, Belgium. ${ }^{4}$ The Global Fund to Fight Aids, Tuberculosis and Malaria, Geneva, Switzerland. ${ }^{5}$ Maugeri Care and Research Institute, Tradate, Italy.

Correspondence: Jose A. Caminero, Pneumology Department, Hospital General de Gran Canaria "Dr. Negrin", Barranco de la Ballena s/n, 35010 Las Palmas de GC, Spain.

E-mail: jcamlundagobiernodecanarias.org

@ERSpublications

A potential standardised regimen to manage extensively drug-resistant tuberculosis (XDR-TB) and pre-XDR cases http://ow.ly/LucH30bUjjH

Cite this article as: Caminero JA, Piubello A, Scardigli A, et al. Proposal for a standardised treatment regimen to manage pre- and extensively drug-resistant tuberculosis cases. Eur Respir J 2017; 50: 1700648 [https://doi.org/10.1183/13993003.00648-2017].

\section{Introduction}

Drug-resistant tuberculosis (TB) has become one of the main obstacles to controlling (and eventually eliminating) this disease [1-4]. The World Health Organization (WHO) reports that the number of patients suffering from TB with resistance to rifampicin (RR-TB) or multidrug-resistant TB (MDR-TB, resistance to at least isoniazid and rifampicin) is increasing alarmingly each year. Furthermore, the treatment success rates achieved globally are suboptimal, barely exceeding $50 \%[1,2,5]$. The proportion decreases to $25 \%$ in patients with extensively drug-resistant tuberculosis (XDR-TB) (MDR-TB plus resistance to at least one fluoroquinolone (FQ) and a second-line injectable (SLI) drug) and to $<20 \%$ when the drug resistance profile is beyond $\operatorname{XDR}[1,2,5]$.

The outcomes observed as of today suggest that specific patients achieve treatment success, but the impact on the RR-/MDR-TB epidemic is rather limited $[6,7]$.

Therefore, the need to improve our capacity to render permanently noninfectious and cure the vast majority of M/XDR-TB patients is obvious [8]. To achieve this goal, the WHO has recently recommended the so-called "shorter regimens" (9-12 months) for patients with RR-/MDR-TB who are susceptible to FQs and SLIs, or who have not previously received these two groups of drugs [2, 9-13].

These "shorter regimens" have achieved relapse-free success rates in $85-90 \%$ of the MDR-TB patients treated [14-17].

While an impact on the MDR-TB epidemic is forecast by scaling-up the use of these regimens, this does not work automatically for XDR- or pre-XDR-TB cases. The success rate in these cases depends on several factors (e.g. challenges to confirm the resistance patterns, availability of new drugs, clinical training, programmatic conditions, etc.). 
The aim of this article is to discuss the possibility of designing a standardised regimen which, ideally supported by the use of rapid diagnostic methods to identify eligible patients [9], would be potentially effective in the majority of cases affected by pre-XDR- and XDR-TB.

However, even without access to these rapid diagnostic methods, or waiting for their results, this regimen could work in patients failing the shorter or conventional standardised MDR-TB regimens. Therefore, the target of this regimen would be most of the patients with pre-XDR- and XDR-TB.

This regimen would complement the existing set of available standard regimens for new cases and MDR-TB cases (the WHO-recommended “shorter" regimen).

\section{Rationale for a standardised regimen to manage pre-XDR-TB and XDR-TB cases}

The first and most important justification to look for a potential standard regimen is represented by the low success and high failure/death rates presently achieved in treating pre-XDR and XDR-TB cases [1, 2, 9, 14].

Moreover, currently many of the XDR-TB cases are patients failing the MDR-TB standardised regimen, or new TB patients who are in contact with these cases. Therefore, Mycobacterium tuberculosis strains in these patients often carry a very similar pattern of extended resistance, including the drugs previously prescribed (ethionamide/prothionamide, cycloserine/terizidone among others) [18-21].

\section{Basic regimen requirements}

This regimen should satisfy four basic requirements, common to all anti-TB regimens [18-20]:

1) at least four new drugs (never used before), likely to be effective, need to be present;

2) two of them should be "core" drugs, at least one having a good bactericidal activity and one a good sterilising activity. Ideally, both (or at least the sterilising drug) should be given for the entire duration of treatment;

3) the other two drugs are the so-called "companion" drugs, whose function is to protect the action of the core drugs;

4) the treatment should be administered for sufficient time to cure patients while preventing relapse. Compounds with higher sterilising activity (rifampicin, pyrazinamide and, probably, moxifloxacin, levofloxacin, linezolid, bedaquiline and delamanid) [19] are potentially useful to reduce the treatment duration, although some of them will not be eligible for this regimen [19, 20].

\section{Regimen composition and possible alternatives}

To be consistent with the regimen requirements discussed above, and with the available drugs' characteristics [9], the following antibiotics are candidates for the proposed standardised regimen:

1) Linezolid. Core drug with bactericidal and sterilising capacity [19, 22-25]. It needs to be prescribed at

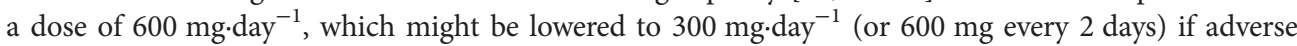
events occur.

2) Bedaquiline. This is also a potentially core drug with bactericidal and sterilising characteristics [18, 19, 26-30]. Some countries may value the use of delamanid instead of bedaquiline, as it also has the features of a core drug $[19,28,31,32]$.

In very selected cases with high level FQ resistance (e.g. mutation in gyaA94Gly) and complete SLI resistance (mutation in rrs1410 or 1484) the possibility of a future combined use of bedaquiline and delamanid might be considered given special criteria are met [33]. This might be the only alternative in those low resource settings where the use of carbapenems is not possible.

3) Clofazimine. It may be considered core because of its possible sterilising capacity [14-17, 19, 34-39], although it can at least play the role of a companion drug. If the patient has previously received clofazimine in a failing regimen ("shorter RR/MDR-TB regimen"), this drug should be replaced by cycloserine (that just plays the role of a companion drug).

4) A carbapenem class antibiotic, which needs to be administered with amoxicillin/clavulanic acid to protect its action. Although it is now a candidate to be a companion drug, there is increasing evidence that it may play a future core drug role [19, 40], especially because of its possible good bactericidal activity. Based on the existing evidence, the best carbapenem would probably be meropenem [41, 42], although imipenem/cilastatin (often more easily available) [42,43] or ertapenem (given the possibility to administer it once a day intramuscularly, it can act as switch therapy useful for ambulatory/home care) might also have a role [44-46]. However, given the need for parenteral administration with carbapenems, delamanid is also a potential choice (needing electrocardiogram monitoring), as discussed previously. Para-amino salicylic acid (PAS), which is a weaker alternative, can be considered only in resource-limited settings under the conditions that LPA (line probe assay) for second-line drugs (SLDs) is available and susceptibility to any FQ and/or any SLI is documented (a combination not often available in real life). 
5) Furthermore, there is need to reinforce and protect the four drugs mentioned above with more "supporting" drugs (important for XDR- and even more for pre-XDR-TB):

a. An FQ, different from that used in the MDR-TB regimen, (e.g. high doses levofloxacin if moxifloxacin has been used previously, and vice versa). In case of pre-XDR-TB because of SLI resistance, this FQ would play a key role in the regimen [19]. But even in cases of XDR-TB, the FQ might still play a role, especially considering that there is no complete cross-resistance among all new FQs [47-50]. If the result of conventional drug susceptibility testing (DST) demonstrates resistance to all FQs, including high concentrations of moxifloxacin, this FQ could be discontinued. If moxifloxacin is used, it is necessary to closely monitor the QTc interval (corrected QT) in the electrocardiogram, because the regimen includes already two other QT-prolonging drugs (bedaquiline and clofazimine), plus the possibility to use delamanid.

b. A SLI different from that used in the MDR-TB regimen. For instance, amikacin can be considered if kanamycin has been used before. However, ideally all these patients should undergo LPA for SLDs to identify correctly the eligible patients based on the mutations identified. The SLI plays an essential role in pre-XDR-TB because of FQ resistance, although its use is still important in cases of XDR-TB, because of the nontotal cross-resistance among FQs described above. However, following demonstration that the mutations rrs1410 and rrs1484 do indeed carry cross-resistance between all SLIs, when these mutations are present the use of streptomycin could be considered in the absence of rpSL mutations [51, 52]. Moreover, if DST shows resistance to all SLIs, they need to be stopped and streptomycin can be considered (given DST susceptibility exists). In cases of complete resistance to all the SLIs and to streptomycin, this drug should be removed from the regimen.

c. High-dose isoniazid may have a role in cases with low-level resistance [53, 54]. It might also be used to treat failures in the shorter MDR-TB regimen, where isoniazid is prescribed at the dose of $10 \mathrm{mg} \cdot \mathrm{kg}^{-1}$ body weight [2]. However, in this XDR-TB regimen, isoniazid would be recommended at daily doses of $15-20 \mathrm{mg} \cdot \mathrm{kg}^{-1}$ body weight. According to the available evidence, isonazid should be discontinued when high-level resistance is confirmed in vitro and when the LPA demonstrates double mutation in kat $G$ and $\operatorname{inh} A$.

As described, this proposed standardised six or seven drug regimen meets the minimum accepted requirements for all TB treatments [18-20]. It includes four new drugs (linezolid, bedaquiline, clofazimine/cycloserine and carbapenem/delamanid) and two or three possible supporting drugs. Of the four new drugs, at least two are bactericidal (linezolid and bedaquiline). Additionally, bactericidal activity may also be ensured by the carbapenem (or delamanid) and by the three supporting drugs (FQ, SLI and high-dose isoniazid) if these drugs are effective on the given strain.

The regimen includes also at least two or three sterilising drugs (linezolid, bedaquiline and clofazimine, if used in the regimen). Furthermore, the sterilising activity of the regimen could be also enhanced by a FQ. Finally, as the regimen includes two or three sterilising drugs, it is possible that in the future (if controlled clinical trials will support) the regimen might be shortened (e.g. 12 months after bacteriological conversion).

A summary of the possibilities to design a standardised regimen for patients with pre-XDR-TB or $\mathrm{XDR}-\mathrm{TB}$ is presented in table 1 . Each country will fine-tune it according to the drugs being used in the $\mathrm{RR} / \mathrm{MDR}-\mathrm{TB}$ regimen and their availability.

The doses recommended for each of these drugs are summarised in table 2.

\section{What patients would be eligible to receive this standardised regimen}

As discussed above, this standardised regimen could target the majority of confirmed pre-XDR-TB or XDR-TB patients in the world. Potentially, the regimen has the characteristics to manage also patients failing a standardised MDR-TB regimen (conventional 21 months or shorter 9-12 months regimens) while waiting for the DST results to SLD. Although LPA and DST testing is strongly recommended, the regimen might be effective also in settings where LPA and DST are eventually not available. However, the patients who have already received more than one of the four new drugs included in the regimen, or those for whom resistances to the core drugs of this regimen has been confirmed with rapid testing, will not be eligible.

\section{Duration of the proposed regimen}

Because of the composition of the proposed regimen (which includes two or three sterilising core drugs), the total duration of the regimen would possibly be $13-15$ months, with at least up to 12 months treatment following culture conversion. All drugs are necessary throughout the treatment (particularly 
TABLE 1 Summary of the possibilities to design a standardised regimen for patients with pre-XDR-TB or XDR-TB

$\begin{array}{lll}\text { Regimen Drug Activity Proposed recommendations } & \text { P }\end{array}$

composition

\begin{tabular}{|c|c|c|c|}
\hline $\begin{array}{l}\text { 1. Two core drugs } \\
\text { (always in the } \\
\text { regimen) }\end{array}$ & Bedaquiline & $\begin{array}{l}\text { Bactericidal and } \\
\text { sterilising } \\
\text { Bactericidal and } \\
\text { sterilising }\end{array}$ & $\begin{array}{l}\text { Delamanid could replace linezolid or bedaquiline if: } \\
\text { one of these drugs has been used previously } \\
\text { proven resistance to one of these drugs } \\
\text { severe toxicity. } \\
\text { Bedaquiline: potentially QT-prolonging drug }\end{array}$ \\
\hline \multirow{2}{*}{$\begin{array}{l}\text { 2.a One } \\
\text { companion } \\
\text { drug } \\
\text { lone of the } \\
\text { following) }\end{array}$} & Clofazimine (first choice) & Sterilising & $\begin{array}{l}\text { To be given if never administered before, especially useful in } \\
\text { MDR-TB patients failing a conventional } 21-24 \text { months MDR-TB } \\
\text { regimen. Potentially QT-prolonging drug }\end{array}$ \\
\hline & Cycloserine & $\begin{array}{l}\text { No bactericidal and } \\
\text { sterilising }\end{array}$ & $\begin{array}{l}\text { To be given if never administered before, and when clofazimine } \\
\text { was administered in a previous failing regimen (shorter 9- } \\
12 \text { months RR/MDR-TB regimen) }\end{array}$ \\
\hline \multirow{3}{*}{$\begin{array}{l}\text { 2.b One } \\
\text { companion } \\
\text { drug } \\
\text { (one of the } \\
\text { followingl }\end{array}$} & $\begin{array}{l}\text { One carbapenem+amoxicillin/ } \\
\text { clavulanate }\end{array}$ & Bactericidal & $\begin{array}{l}3 \text { options: } \\
\text { meropenem (first choice) } \\
\text { imipenem/cilastatin } \\
\text { ertapenem }\end{array}$ \\
\hline & Delamanid & $\begin{array}{l}\text { Bactericidal and } \\
\text { sterilising }\end{array}$ & ECG monitoring needed \\
\hline & $\begin{array}{l}\text { PAS } \\
\text { (para-aminosalicylic acid) }\end{array}$ & Bacteriostatic & $\begin{array}{l}\text { Justified in low income countries under the following conditions: } \\
\text { carbapenems and delamanid cannot be used } \\
\text { LPA to SLD showing possible susceptibility to Amk or Mfx }\end{array}$ \\
\hline \multirow{5}{*}{$\begin{array}{l}\text { 3. Three } \\
\text { supporting } \\
\text { drugs } \\
\text { One FQ } \\
\text { One SLI } \\
\text { High-dose } \\
\text { isoniazid }\end{array}$} & $\begin{array}{l}\text { One FQ } \\
\text { (moxifloxacin) }\end{array}$ & $\begin{array}{l}\text { Bactericidal and } \\
\text { sterilising }\end{array}$ & $\begin{array}{l}\text { Depending on the patient's previous treatment history } \\
\text { In pre-XDR or XDR-TB patients not previously treated for TB } \\
\text { with a FQ } \\
\text { QTc interval close monitoring if used with bedaquiline and } \\
\text { clofazimine }\end{array}$ \\
\hline & $\begin{array}{l}\text { One FQ } \\
\text { (high dose moxifloxacin) }\end{array}$ & $\begin{array}{l}\text { Bactericidal and } \\
\text { sterilising }\end{array}$ & If levofloxacin has been used previously to treat TB \\
\hline & $\begin{array}{l}\text { One FQ } \\
\text { (high dose levofloxacin) } \\
\text { If a DST shows resistance to le }\end{array}$ & $\begin{array}{l}\text { Bactericidal and } \\
\text { sterilising } \\
\text { ofloxacin and moxiflo }\end{array}$ & If moxifloxacin has been used previously to treat TB \\
\hline & $\begin{array}{l}\text { One second line injectable } \\
\text { (amikacin) }\end{array}$ & Bactericidal & $\begin{array}{l}\text { In pre-XDR or XDR-TB patient not previously treated for TB with } \\
\text { a SLI } \\
\text { If kanamycin or capreomycin have been used previously to } \\
\text { treat TB }\end{array}$ \\
\hline & $\begin{array}{l}\text { If a DST shows resistance to all } \\
\text { High dose isoniazid }\end{array}$ & $\begin{array}{l}\text { the second line injec } \\
\text { Bactericidal }\end{array}$ & $\begin{array}{l}\text { Dbles, this drug will be discontinued from the regimen } \\
\text { Dose: } 15-20 \mathrm{mg} \cdot \mathrm{kg}^{-1} \\
\text { Isonazid is discontinued when: } \\
\text { high level of resistance is confirmed in vitro } \\
\text { when the LPA demonstrates double mutation in katG and ir }\end{array}$ \\
\hline
\end{tabular}

XDR-TB: extensively drug-resistant tuberculosis; QT: measure of the time between the start of the $Q$ wave and the end of the $T$ wave in the heart's electrical cycle; MDR-TB: multidrug-resistant tuberculosis; RR: rifampicin-resistance; ECG: electrocardiogram; LPA: Line Probe Assay; Amk: amikacin; Mfx: moxifloxacin; SLD: second-line drug: FQ: fluoroquinolone; QTc: corrected QT; SLI: second-line injectables.

those which are new and core, such as linezolid and bedaquiline), with the exception of SLI and high-dose isoniazid, which could be discontinued after 4 months of treatment. However, these drugs could be extended to 6 months if sputum smear microscopy is positive at the end of the fourth month of treatment. If sputum smear microscopy and culture remain positive at the end of the sixth month of treatment, a regimen failure should be accepted and the case should be re-evaluated to undergo an individualised regimen. If a carbapenem is included in the regimen, it might be discontinued after 6 months following bacteriological conversion. As bedaquiline is a core drug in this regimen, it should be administered for the whole treatment duration (not only for 6 months) [2], as it already happens in some settings $[30,55,56]$.

\section{Possible severe adverse effects and controls throughout treatment}

The adverse events are likely to be similar to those occurring with the regimen currently recommended for the treatment of MDR-TB, including shorter regimens. A potential advantage (which of course needs to be confirmed by adequate studies) is that the potential new regimen contains fewer drugs of most of the anti-XDR regimens currently in use. 


\begin{tabular}{|c|c|c|c|c|c|}
\hline \multirow[t]{2}{*}{ Drug } & \multicolumn{5}{|c|}{ Body weight kg } \\
\hline & $30-35$ & $36-45$ & $46-55$ & $56-70$ & $>70$ \\
\hline Linezolid $600 \mathrm{mg}$ & 1 pill & 1 pill & 1 pill & 1 pill & 1 pill \\
\hline Bedaquiline $100 \mathrm{mg}$ & \multicolumn{5}{|c|}{$\begin{array}{l}4 \text { pills } / 24 \mathrm{~h} \text { during } 2 \text { weeks, followed by } 2 \text { pills } 3 \text { times weekly } \\
\text { until the end of the treatment }\end{array}$} \\
\hline Delamanid 50 mg & \multicolumn{5}{|c|}{2 pills $/ 12 \mathrm{~h}$} \\
\hline Clofazimine $100 \mathrm{mg}$ & \multicolumn{5}{|c|}{1 pill } \\
\hline Meropenem/imipenem & $500 \mathrm{mg} / 12 \mathrm{~h}$ & $500 \mathrm{mg} / 12 \mathrm{~h}$ & $1 \mathrm{gr} / 12 \mathrm{~h}$ & $1 \mathrm{gr} / 12 \mathrm{~h}$ & $1,5 \mathrm{gr} / 12 \mathrm{~h}$ \\
\hline Amoxicillin/clavulanate & \multicolumn{5}{|c|}{$1000 \mathrm{mg} / 12 \mathrm{~h}$} \\
\hline Amikacin/kanamycin/capreomycin & $0.5 \mathrm{~g}$ & $0.750 \mathrm{~g}$ & $0.750 \mathrm{~g}$ & $1 \mathrm{~g}$ & $1 \mathrm{~g}$ \\
\hline Moxifloxacin & $400 \mathrm{mg}$ & $600 \mathrm{mg}$ & $600 \mathrm{mg}$ & $800 \mathrm{mg}$ & $800 \mathrm{mg}$ \\
\hline Levofloxacin & $500 \mathrm{mg}$ & $750 \mathrm{mg}$ & $1 \mathrm{gr}$ & $1 \mathrm{gr}$ & $1 \mathrm{gr}$ \\
\hline PAS $4 \mathrm{~g}$ & $1+1$ pill & $1+1$ pill & $1+1$ pill & $1+1$ pill & $1+1+1$ pill \\
\hline Isoniazid 300 mg & 2 pills & 2.5 pills & 3 pills & 3.5 pills & 4 pills \\
\hline
\end{tabular}

XDR-TB: extensively drug-resistant tuberculosis; PAS: para-aminosalicylic acid.

Close attention is necessary on possible electrocardiogram (ECG) alterations (QT interval), as several QTc-prolonging drugs are part of the possible new regimen (bedaquiline, sometimes delamanid, clofazimine and FQs, especially if moxifloxacin is used). A weekly ECG control will be necessary at least in the first month of treatment; it can eventually be reduced in frequency (e.g. monthly) in the absence of clinical disturbances or previous QTc increase over $500 \mathrm{~ms}$.

Other adverse events are likely to occur, including optic neuritis, or haematological alterations (usually due to linezolid). The reduction of the linezolid dose from 600 to $300 \mathrm{mg} \cdot \mathrm{day}^{-1}$ or $600 \mathrm{mg}$ three times weekly is generally sufficient to manage them $[19,57,58]$. The possibility to use TDM (treatment drug monitoring) to adjust the dose and minimise adverse events has been recently recommended [59, 60].

Therefore, it will be important to monitor these potential adverse effects, e.g. performing a clinical control, blood analysis (including haemogram and biochemistry with liver, renal and pancreatic function tests) and a sputum examination (for bacilloscopy and culture) on a monthly basis. It would also be necessary to perform a monthly audiometry while prescribing SLIs. Finally, a chest radiograph every 3-6 months and, eventually, pregnancy and HIV tests at the start of treatment should be performed.

\section{Potential limitations and risks related to the use of the proposed regimen for pre-XDR- and XDR-TB cases}

As discussed above, the ideal approach is to prescribe the regimen based on GenoType for SLD. However, this regimen has characteristic making it potentially effective in patients failing the standardised MDR-TB regimens, or waiting for the conventional DST result (or, as the extreme scenario, in patients living where these tests are not available). As the potential efficacy of this regimen is based on drugs like linezolid and bedaquiline, the capacity of monitoring their possible toxicity needs to be ensured (especially QTc, haemogram auditory and visual acuity).

Last, but not least, it is imperative that all the drugs composing the regimen are available in the country, that active drug safety monitoring and management (aDSM) is in place, as well as patient-centred and supportive models of care to ensure proper follow-up and care of patients undergoing treatment.

\section{Need for further research to validate this proposal for pre-XDR and XDR-TB cases}

To validate this proposal further clinical and operational research are necessary, including:

1) randomised clinical trial(s) testing this regimen under operational conditions, although designing and conducting them will be challenging. The trial(s) should ideally be able to study the efficacy of the regimen even in patients with TB resistant to FQs and SLIs, and evaluate its ideal duration; 
TABLE 3 The three standardised regimens potentially able to manage the different categories of TB cases

TB patients categories

\section{Standardised treatment Recommendations} regimen

$2 \mathrm{HRZE}^{\#} / 4 \mathrm{HR}$

Standardised treatment regimen for patients with

rifampicin-susceptible TB

\section{Standardised treatment regimen for RR/MDR-TB patients,} susceptible to FQs and SLIs

\author{
$4 \mathrm{Km}-\mathrm{hMfx}{ }^{\mathrm{q}}-\mathrm{Cfz}-\mathrm{Eto} /$ \\ Pto-Z-E-hH ${ }^{+} / 5$ \\ $h M f x$ "l-Cfz-E-Z
}
\#: In case of a positive sputum smear microscopy at the end of the second month of treatment, consider to check susceptibility to isoniazid before switching to the continuation phase lisoniazid, rifampicin)
Standardised shortened treatment with SLD
The intensive phase should be extended to 6 months when at the end of the fourth month of treatment, sputum smear microscopy is still positive
ๆ: HMfx $\left(600-800 \mathrm{mg} \cdot \mathrm{day}^{-1}\right)$
+ : $\mathrm{hH}\left(15 \mathrm{mg} \cdot \mathrm{kg}^{-1}\right.$ body weight)
$\S$ : With the considerations on duration and possible change of drugs exposed in the text

\section{Standardised treatment regimen for patients with pre-XDR-TB and XDR-TB
Lzd-Bdq-Mrp/Clav lor $\mathrm{D}(\mathrm{m})-\mathrm{Cfz}-$ $A m k^{\S}-h L f x^{\S}-h H^{\S}$

TB: tuberculosis; H: isoniazid; R: rifampicin; Z: pyrazinamide; E: ethambutol; RR: rifampicin-resistant; MDR: multidrug-resistant; $\mathrm{Km}$ : kanamycin; hMfx: high-dose moxifloxacin; Cfz: clofazimine: Eto: ethionamide; Pto: prothionamide; $\mathrm{hH}$ : high-dose isoniazid; SLD: second-line drug; XDR: extensively drug-resistant; Lzd: linezolid; Bdq: bedaquiline; Mrp/Clav: meropenem/clavulanate; DIm: delamanid; Amk: amikacin; hLfx: high-dose levofloxacin.

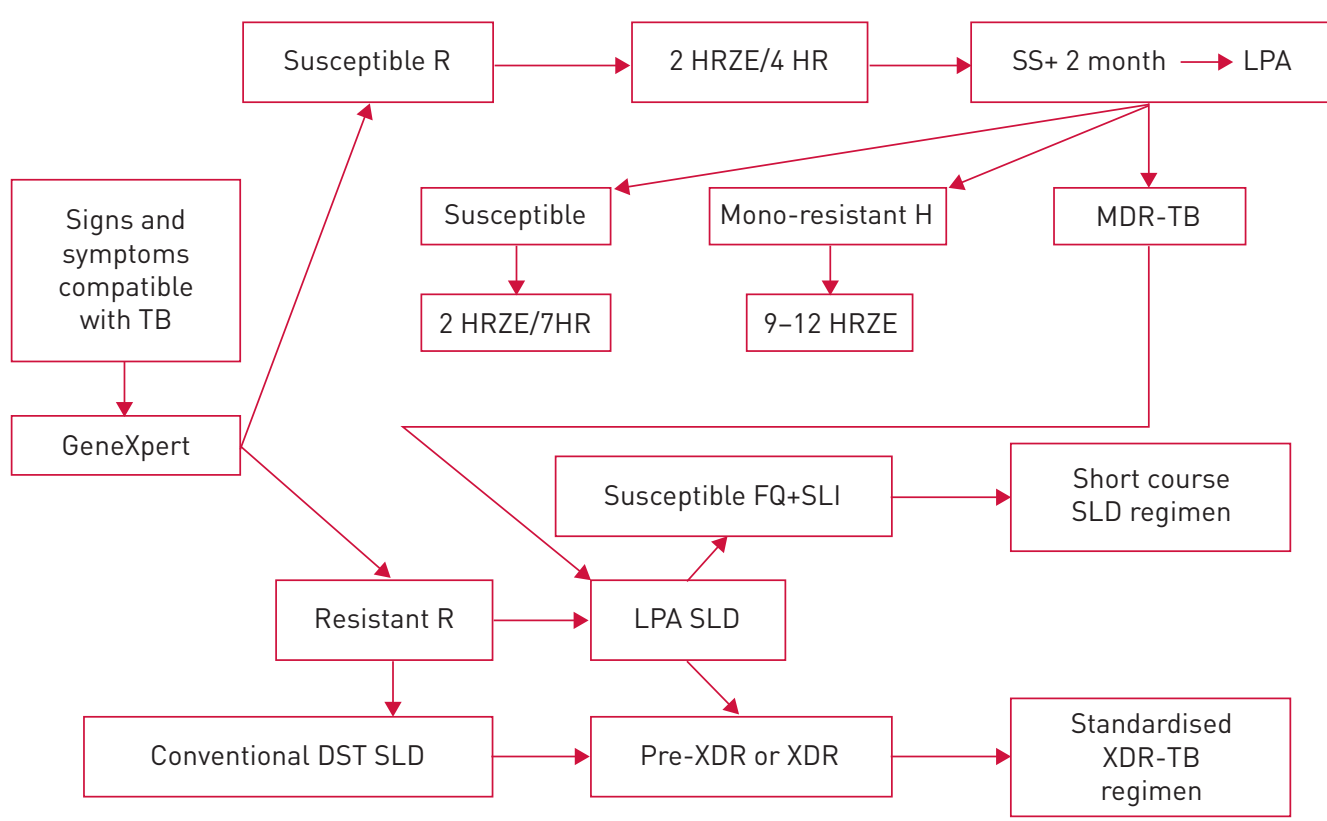

FIGURE 1 Proposed algorithm ensuring early detection of tuberculosis and prevention of possible amplification of the resistance pattern in programmatic conditions. R: Rifampicin; H: isoniazid; $\mathrm{Z}$ : pyrazinamide; E: ethambutol; SS+: sputum smear positive; LPA: line probe assay; MDR-TB: multidrug-resistant tuberculosis; XDR-TB: extensively drug-resistant tuberculosis; FQ: fluorquinolones; SLI: second-line injectables; SLD: second-line drugs; DST: drug susceptibility testing. 
2) more evidence on bedaquiline and delamanid safety when used for $>6$ months and programmatically [61] is necessary, as these two drugs are core pillars of the proposed regimen;

3) evidence on safety and efficacy of bedaquiline and delamanid combination is also necessary;

4) finally, the different programmatic and operational aspects of using a similar regimen need to be properly studied.

\section{Proposal to manage most TB patients programmatically using just three standardised regimens}

This article provides the rationale to treat most of the existing TB cases using just three standardised regimens. A summary of the three standardised regimens is available in table 3.

This proposal implies a molecular test performed at the beginning of the treatment to ensure susceptibility to rifampicin (GeneXpert and/or GenoType). If the strain is susceptible to rifampicin, the standardised first-line drug regimen (2HRZE/4HR, 2 months with isoniazid, rifampicin, pyrazinamide and ethambutol followed by 2 months with isoniazid and rifampicin) can be administered. On the contrary, if RR/ MDR-TB is detected, a GenoType for FQs and SLIs (second-line injectables) needs to be performed. If this RR/MDR-TB strain is susceptible to FQs and SLIs, the standardised shorter 9-12 months RR/ MDR-TB regimen should be offered to the patient. But if GenoType shows resistance to FQs and/or SLIs, the standardised regimen proposed in this article can be potentially considered.

Figure 1 shows the possible decision tree supporting this proposal.

\section{Conclusions}

In this manuscript we have proposed and discussed the rationale for proposing a standardised regimen to manage most of the patients with pre-XDR- and XDR-TB. We have also discussed the potential advantages and threats, which need to be confirmed by adequate studies.

If future studies will demonstrate that this approach is effective and safe, three standardised regimens will be potentially able to manage an important proportion of the existing TB patients (susceptible, MDR- and pre-XDR/XDR-TB ones) under programmatic conditions (table 3 and figure 1).

\section{Acknowledgements}

The authors alone are responsible for the views expressed in this article and they do not necessarily represent the decisions and policies of their institutions.

The authors wish to thank Lia D'Ambrosio and Rosella Centis (Maugeri Care and Research Institute, Tradate, Italy) for their useful comments and the support in preparing the manuscript.

\section{References}

1 World Health Organization. Global tuberculosis report 2015. Document WHO/HTM/TB/2015. Geneva, World Health Organization, 2015.

2 World Health Organization. Global tuberculosis report 2016. Document WHO/HTM/TB/2016. Geneva, World Health Organization, 2016.

3 Lönnroth K, Migliori GB, Abubakar I, et al. Towards tuberculosis elimination: an action framework for low-incidence countries. Eur Respir J 2015; 45: 928-952.

4 D’Ambrosio L, Dara M, Tadolini M, et al. Tuberculosis elimination: theory and practice in Europe. Eur Respir J 2014; 43: 1410-1420.

5 Migliori GB, Sotgiu G, Gandhi NR, et al. Drug resistance beyond extensively drug-resistant tuberculosis: individual patient data meta-analysis. Eur Respir J 2013; 42: 169-179.

6 Kendall EA, Fojo AT, Dowdy DW. Expected effects of adopting a 9 month regimen for multidrug-resistant tuberculosis: a population modelling analysis. Lancet Respir Med 2017; 5: 191-199.

7 Sotgiu G, Migliori GB. Effect of the short-course regimen on the global epidemic of multidrug-resistant tuberculosis. Lancet Respir Med 2017; 5: 159-161.

8 Grzybowski S, Enarson DA. The fate of cases of pulmonary tuberculosis under various treatment programmes. Bull Int Union Tuberc Lung Dis 1978; 53: 70-75.

9 Falzon D, Schünemann HJ, Harausz E, et al. WHO treatment guidelines for drug-resistant tuberculosis, 2016 update. Eur Respir J 2017; 49: 1602308.

10 Sotgiu G, Tiberi S, D'Ambrosio L, et al. Faster for less, the new 'Shorter' regimen for multidrug-resistant tuberculosis. Eur Respir J 2016; 48: 1503-1507.

11 van der Werf MJ, Hollo V, Ködmön C, et al. Eligibility for shorter treatment of multidrug-resistant tuberculosis in the European Union. Eur Respir J 2017; 49: 1601992.

12 Javaid A, Ahmad N, Khan AH, et al. Applicability of World Health Organization's recommended new shorter regimen in a multidrug-resistant tuberculosis high burden country. Eur Respir J 2017; 49: 1601967.

13 Dalcolmo M, Gayoso R, Sotgiu G, et al. Resistance profile to the drugs composing the 'shorter' regimen for multidrug-resistant TB in Brazil, 2000-2015. Eur Respir J 2017; 49: 1602309.

14 Van Deun A, Aung KJM, Halim MA, et al. Short, highly effective, and inexpensive standardized treatment of multidrug-resistant tuberculosis. Am J Respir Crit Care Med 2010; 182: 684-692. 
15 Aung KJM, Van Deun A, Declercq E, et al. Successful '9-month Bangladesh regimen' for multidrug-resistant tuberculosis among over 500 consecutive patients. Int J Tuberc Lung Dis 2014; 18: 1180-1187.

16 Piubello A, Hassane Harouna S, Souleymane MB, et al. High cure rate with standardised short-course multidrug-resistant tuberculosis treatment in Niger: no relapses. Int J Tuberc Lung Dis 2014; 18: 1188-1194.

17 Kuaban C, Noeske J, Rieder HL, et al. High effectiveness of a 12-month regimen for MDR-TB patients in Cameroon. Int J Tuberc Lung Dis 2015; 19: 517-524.

18 Caminero JA, Matteelli A, Lange C. Treatment of TB. In: Lange C, Migliori GB, eds. Tuberculosis (ERS Monograph). Sheffield, European Respiratory Society, 2012; pp. 154-166.

19 Caminero JA, Scardigli A. Classification of antituberculosis drugs: a new proposal based on the most recent evidence. Eur Respir J 2015; 46: 887-893.

20 Caminero JA, Van Deun A, Fujiwara PI, et al. Guidelines for clinical and operational management of drug-resistant tuberculosis. Paris, International Union Against Tuberculosis and Lung Disease, 2013.

21 Scardigli A, Caminero JA, Sotgiu G, et al. Efficacy and tolerability of ethionamide versus prothionamide: a systematic review. Eur Respir J 2016; 48: 946-952.

22 Dietze R, Hadad DJ, McGee B, et al. Early and extended bactericidal activity of linezolid in pulmonary tuberculosis. Am J Respir Crit Care Med 2008; 178: 1180-1185.

23 Sotgiu G, Centis R, D'Ambrosio L, et al. Linezolid to treat extensively drug-resistant TB: retrospective data are confirmed by experimental evidence. Eur Respir J 2013; 42: 288-290.

24 Tang S, Yao L, Hao X, et al. Efficacy, safety and tolerability of linezolid for the treatment of XDR-TB: a study in China. Eur Respir J 2015; 45: 161-170.

25 Lee M, Lee J, Carroll MW, et al. Linezolid for treatment of chronic extensively drug-resistant tuberculosis. $N$ Engl J Med 2012; 367: 1508-1518.

26 Rustomjee R, Diacon AH, Allen J, et al. Early bactericidal activity and pharmacokinetics of the diarylquinoline TMC207 in treatment of pulmonary tuberculosis. Antimicrob Agents Chemother 2008; 52: 2831-2835.

27 Diacon AH, Pym A, Grobusch MP, et al. Multidrug-resistant tuberculosis and culture conversion with bedaquiline. $N$ Engl J Med 2014; 371: 723-732.

28 Brigden G, Hewison C, Varaine F. New developments in the treatment of drug-resistant tuberculosis: clinical utility of bedaquiline and delamanid. Infect Drug Resistance 2015; 8: 367-378.

29 Pym AS, Diacon AH, Tang SJ, et al. Bedaquiline in the treatment of multidrug- and extensively drug-resistant tuberculosis. Eur Respir J 2016; 47: 564-674.

30 Ndjeka N, Conradie F, Schnippel K, et al. Treatment of drug-resistant tuberculosis with bedaquiline in a high HIV prevalence setting: an interim cohort analysis. Int J Tuberc Lung Dis 2015; 19: 979-985.

31 Diacon AH, Dawson R, Hanekom M, et al. Early bactericidal activity of delamanid (OPC-67683) in smear-positive pulmonary tuberculosis patients. Int J Tuberc Lung Dis 2011; 15: 949-954.

32 Skipconoka V, Danilovits M, Pehme L, et al. Delamanid improves outcomes and reduces mortality in multidrug-resistant tuberculosis. Eur Respir J 2013; 41: 1393-1400.

33 Matteelli A, D'Ambrosio L, Centis R, et al. Compassionate and optimum use of new tuberculosis drugs. Lancet Infect Dis 2015; 15: 1131-1132.

34 Williams K, Minkowski A, Amoabeng O, et al. Sterilizing activities of novel combinations lacking first- and second-line drugs in a murine model of tuberculosis. Antimicrob Agents Chemother 2012; 56: 3114-3120.

35 Padayatchi N, Gopal M, Naidoo R, et al. Clofazimine in the treatment of extensively drug-resistant tuberculosis with HIV coinfection in South Africa: a retrospective cohort study. J Antimicrob Chemother 2014; 69: 3103-3107.

36 Tyagi S, Ammerman NC, Li SY, et al. Clofazimine shortens the duration of the first-line treatment regimen for experimental chemotherapy of tuberculosis. Proc Natl Acad Sci USA 2015; 112: 869-874.

37 Diacon AH, Dawson R, von Groote-Bidlingmaier F, et al. Bactericidal activity of pyrazinamide and clofazimine alone and in combinations with pretomanid and bedaquiline. Am J Respir Crit Care Med 2015; 191: 943-953.

38 Tang S, Yao L, Hao X, et al. Clofazimine for the treatment of multidrug-resistant tuberculosis: prospective, multicenter, randomized controlled study in China. Clin Infect Dis 2015; 60: 1361-1367.

39 Dalcolmo M, Gayoso R, Sotgiu G, et al. Effectiveness and safety of clofazimine within a standard multidrug-resistant tuberculosis regimen in Brazil: first nation-wide report on over 2,500 cases. Eur Respir J 2017; 49: 1602445.

40 De Lorenzo S, Alffenaar JW, Sotgiu G, et al. Efficacy and safety of meropenem-clavulanate added to linezolid-containing regimens in the treatment of MDR-/XDR-TB. Eur Respir J 2013; 41: 1386-1392.

41 Tiberi S, Payen MC, Sotgiu G, et al. Effectiveness and safety of meropenem/clavulanate-containing regimens in the treatment of MDR- and XDR-TB. Eur Respir J 2016; 47: 1235-1243.

42 Tiberi S, Sotgiu G, D'Ambrosio L, et al. Comparison of effectiveness and safety of imipenem/clavulanate- versus meropenem/clavulanate-containing regimens in the treatment of MDR- and XDR-TB. Eur Respir J 2016; 47: 1758-1766.

43 Tiberi S, Sotgiu G, D'Ambrosio L, et al. Effectiveness and safety of imipenem-clavulanate added to an optimized background regimen (OBR) versus OBR control regimens in the treatment of multidrug-resistant and extensively drug-resistant tuberculosis. Clin Infect Dis 2016; 62: 1188-1190.

44 van Rijn SP, van Altena R, Akkerman OW, et al. Pharmacokinetics of ertapenem in patients with multidrug-resistant tuberculosis. Eur Respir J 2016; 47: 1229-1234.

45 Srivastava S, van Rijn SP, Wessels AMA, et al. Susceptibility testing of antibiotics that degrade faster than the doubling time of slow-growing mycobacteria: ertapenem sterilizing effect versus Mycobacterium tuberculosis. Antimicrob Agents Chemother 2016; 60: 3193-3195.

46 Tiberi S, D'Ambrosio L, De Lorenzo S, et al. Ertapenem in the treatment of multidrug-resistant tuberculosis: first clinical experience. Eur Respir J 2016; 47: 333-336.

47 Jo KW, Lee SD, Kim WS, et al. Treatment outcomes and moxifloxacin susceptibility in ofloxacin-resistant multidrug-resistant tuberculosis. Int J Tuberc Lung Dis 2014; 18: 39-43.

48 Cheng AFB, Yew WW, Chan EWC, et al. Multiplex PCR amplimer conformation analysis for rapid detection of gyrA mutations in fluoroquinolone-resistant Mycobacterium tuberculosis clinical isolates. Antimicrob Agents Chemother 2004; 48: 596-601.

49 Zignol M, Dean AS, Alikhanova N, et al. Population-based resistance of Mycobacterium tuberculosis isolates to pyrazinamide and fluoroquinolones: results from a multicountry surveillance project. Lancet Infect Dis 2016; 16: $1185-1192$. 
50 Wang JY, Lee LN, Lai HC, et al. Fluoroquinolone resistance in Mycobacterium tuberculosis isolates: associated genetic mutations and relationship to antimicrobial exposure. J Antimicrob Chemother 2007; 59: 860-865.

51 Caminero JA, Sotgiu G, Zumla A, et al. Best drug treatment for multidrug-resistant and extensively drug-resistant tuberculosis. Lancet Infect Dis 2010; 10: 621-629.

52 Georghiou SB, Magana M, Garfein RS, et al. Evaluation of genetic mutations associated with Mycobacterium tuberculosis resistance to amikacin, kanamycin and capreomycin: a systematic review. PLoS ONE 2012; 7: e33275.

53 Katiyar SK, Bihari S, Prakash S, et al. A randomised controlled trial of high-dose isoniazid adjuvant therapy for multidrug-resistant tuberculosis. Int J Tuberc Lung Dis 2008; 12: 129-145.

54 Cambau E, Viveiros M, Machado D, et al. Revisiting susceptibility testing in MDR-TB by a standardized quantitative phenotypic assessment in a European multicentre study. J Antimicrob Chemother 2015; 70: 686-696.

55 Guglielmetti L, Le Dû D, Jachym M, et al. Compassionate use of bedaquiline for the treatment of multidrug-resistant and extensively drug-resistant tuberculosis: interim analysis of a French cohort. Clin Infect Dis 2015; 60: 188-194

56 Lewis JM, Hine P, Walker J, et al. First experience of effectiveness and safety of bedaquiline for 18 months within an optimised regimen for XDR-TB. Eur Respir J 2016; 47: 1581-1584.

57 Sotgiu G, Centis R, D'Ambrosio L, et al. Low minimal inhibitory concentrations of linezolid against multidrug-resistant tuberculosis strains. Eur Respir J 2015; 45: 287-289.

58 Bolhuis MS, Tiberi S, Sotgiu G, et al. Linezolid tolerability in multidrug-resistant tuberculosis: a retrospective study. Eur Respir J 2015; 46: 1205-1207.

59 Nahid P, Dorman SE, Alipanah N, et al. Executive summary: official American Thoracic Society/Centers for Disease Control and Prevention/Infectious Diseases Society of America clinical practice guidelines: treatment of drug-susceptible tuberculosis. Clin Infect Dis 2016; 63: 853-867.

60 Sotgiu G, Nahid P, Loddenkemper R, et al. The ERS-endorsed official ATS/CDC/IDSA clinical practice guidelines on treatment of drug-susceptible tuberculosis. Eur Respir J 2016; 48: 963-971.

61 Borisov SE, Dheda K, Enwerem M, et al. Effectiveness and safety of bedaquiline-containing regimens in the treatment of multidrug and extensively drug-resistant tuberculosis: a multicentre study. Eur Respir J 2017; 49: 1700387. 